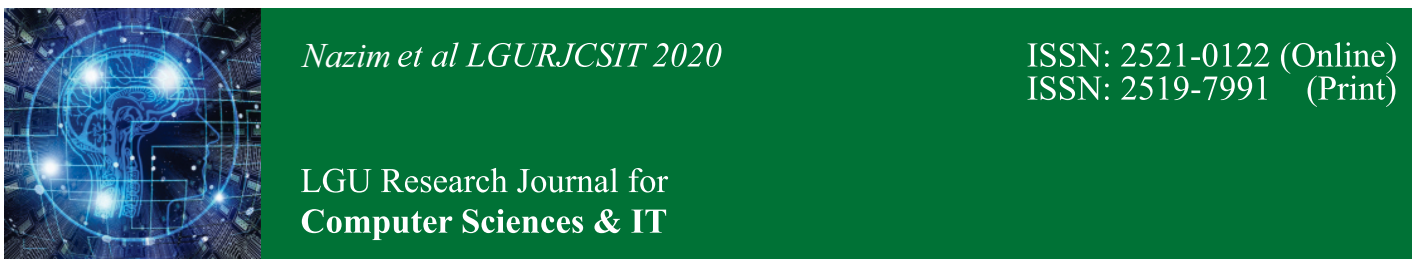

Vol. 4 Issue 1, Jan. - March 2020

\title{
Requirement Gathering Techniques Widely Used in Global Software Engineering: A Comprehensive Study \\ Zarsha Nazim ${ }^{1}$, Mubashir Ali ${ }^{2}$, Ayesha Ajmal ${ }^{3}$ \\ ${ }^{1,3}$ Department of Software Engineering, Forman Christian College, Lahore, Pakistan \\ ${ }^{2}$ Department of Computer Science, Bahauddin Zakariya University, Multan, Pakistan; \\ ²dr.mubashirali1@gmail.com
}

\begin{abstract}
:
This research paper is solely based upon conducting the study with respect to analysis of the recent work done in the field of Global Software Engineering (GSE). It is massively spreading within various organizations. GSD is significant with respect to cost reduction, efficiency in context of delivery of software and extending economic resources. The working model of GSE is a complex and there are certain threats that are directly targeting the audiences. The factors affecting are the differences in context of Culture and geographic distances that can directly affect the performance of a software process. One of the important aspects that is kept in consideration in Global Requirement Engineering is particularly communication. The issues with respect to communication can be divided further into the team and targeted clients. The study is highlighting the concept is to generate the best practices in Global Software Engineering (GSE) along with Lexicon model. The research has focused on more than one technique along with demonstrating their advantages to present a solution and a technique that can be adopted to generate requirements in GSE.
\end{abstract}

Keywords: Global Software Engineering, Requirement Gathering Techniques, Natural Language, Lexicon Model

\section{INTRODUCTION}

Requirement gathering is known as a major task in developing a project in global organizations. Requirement Engineering becoming a key challenge in global software engineering [1]. Mostly if problem arises in the functionality of the software or in any phase of the developing cycle of the project, it is due to the fault in requirements gathering or understanding it; or mapping the described problem according to its solution [2].

The qualitative analysis elaborate one of the problems that chosen and by applying a search process some solutions, methodologies, tools and techniques will be extracted to solve the below mentioned problem. Global Software Development (GSD) has distinct features comparatively to any other simple software development thus requirement engineering is the main component for software development [3]. In GSD some issue arises such as language

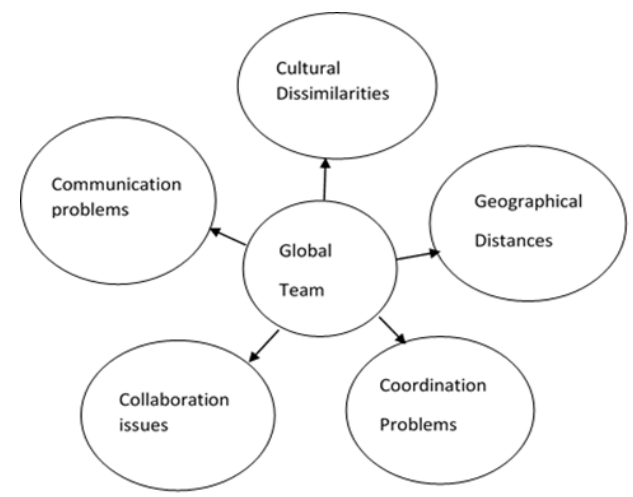

Figure 1: Components of Global Software Engineering

issues, communication issues between client and developer, different culture and such other factors. These problem impacts at the time of requirement gathering. So, project developer uses natural languages model to overcome these problems [4]. Global Requirement Engineering (GRE) includes implementation of product 
along with its design and validation in context to the software products including distinctive functions of time, distance and differences. Regarding the need for conducting a systematic literature review, the research question for this study set to be as follow:

RQ: Which requirement gathering techniques are widely used in Global Software Engineering?

GSD is a modern program in field of software engineering and has a vast industrial scope. The teams are distributed nationally over massive distances. Project Planning is a must along with resource allocation. Many problems are caused by long distances in context to team collaboration over a project. This could be better solved through global software development.

Mostly if problem arises in the functionality of the software or in any phase of the developing cycle of the project, it is due to the fault in requirements gathering or understanding it or mapping the described problem according to its solution. In this survey one of the problems that has been described below has been chosen and by applying a search process some solutions, methodologies, tools and techniques will be extracted to solve the below mentioned problem. Global Software development (GSD) has distinct features comparatively to any other simple software development thus Requirement engineering is the main component for software development. In GSD some issue arises such as language issues, communication issues between client and developer, different culture and such other factors. These problem impacts at the time of requirement gathering

\section{LITERATURE REVIEW}

According to the recent studies, the work done in GRE is comparatively lesser and there has been fewer contributions in this regard [5]. Organizations did not work on adopting practices related to GRE environment. Based on the study, it was suggested that analysis should be conducted to propose a better framework for getting familiar with a context to understand the process of requirements in Global requirement engineering [6]. The major element of requirement engineering is the requirement elicitation and it has to be focused more [7]. The work in this field is slightly lesser and according to the feedback and suggestions of practitioner and the ones who have worked on the literature review of the GRE. It is suggested that after the interview session with the audiences, document analysis has to be taken in consideration to find out the framework for requirement elicitation [8]. After that results should be compared and modelling of dimensions of elicitations needs to be figured out to conduct a survey and validate the model at last.

\subsection{The Project Team Elected A Project Leader}

By using this technique, only one member of the project is not responsible about gather requirements because in this technique, leader took the responsibility of verifying and validating the requirements and information rather than designing the system [9]. Leader will gather the requirements on a global scope for the developers and made them understand how they should develop the system [10]. Choosing a leader for the project management is the major task and the leader should possess the following techniques:
i. Good listener
ii. Best communicator
iii. Have all qualities of leadership

\subsection{Video Conferencing}

Both the project team and the stake holders gathered to join the video conferencing to talk in details about their requirements for the project [11]. Video conferencing is mainly used in the global requirements engineering because both the parties have to discuss in detail and this technique helps them to achieve their goals [12]. In this phase, project team is divided into two shifts, the morning and the evening, and the video conferencing is also established in both the shifts [13]. This helps both the members of the shifts to collaborate with each other and make each other understand what they have gathered and which functionality client requires from them. Drawback of this technique:

i. Every member takes requirements according to its perspective

ii. Lots of conflicts occurs

On the other hand, this technique has also its benefits and these are that if one member could not able to understand the requirements 
the other will help him this decreases the rate of risk and increases the efficiency of the understanding of the requirements [14].

\subsection{In Case of Virtual Teams}

When working in global virtual teams, the process of communication has high influenced in RE. In these scenarios, interviews are considered to be best suited to gather requirements and engage the clients [15]. To collect data in these scenarios, the methodology used is taped in-depth interviews which lasted for 1.5 to 4 hours. The questions were openended and team members have freedom to describe their experiences and problems beyond the questions' boundaries. Follow-up emails and telephone calls after the interviews provided clarification when necessary [16]. Due to this technique, developer get feedback on target project and does system design accordingly. Due to this we can judge market trends and scope of global needs [17].

\subsection{Facilitated Sessions}

In this methodology a large group of five or more members gathered for common purpose. In this session members tried to gather common requirements in the form of group and this technique is considered to be more efficient than conducting interviews. Major benefit of this methodology is that it saves time [18].

\subsection{Designing Interviews}

An interview is the common method used by the requirement engineers to elicit requirements and make objectives of the system clear through verbal conversation with the stakeholders. Interviews could be structured or closed i.e., in the form of predefined questions, semi structured i.e., a blend of predefine and unplanned questions and unstructured or open i.e., an informal interview that does not involve predefined questions. The first two approaches aim towards acquiring quantitative data, whereas research is based on qualitative data and focus is on unplanned and unstructured questions.

\subsection{Domain Analysis}

Domain analysis is used to gather early requirements and capture domain knowledge by deeply observing the existing applications and related documentation. Usually this technique is used by the domain experts to study the domain area thoroughly [18]. This technique is helpful in eliciting requirement from documents, instruction manuals, templates and forms used in the existing system or in the current business processes. Domain analysis also comprises of the domain knowledge of the system that has to be developed and its reusable concepts and components. Mostly, this technique is used when project involves replacement or enhancement in the existing legacy system [19].

\subsection{Scenarios}

Scenarios are mostly used to collect data; it helps developers to understand the problem more effectively and it also decreases the ambiguity in the requirement gathering phase. Scenarios are behavioral descriptions in the context. It defines that what actions perform by actors to achieve a specific goal [20]. It also defines exception case when failure condition happens in the system. The context of scenarios defined by means of geographical location, a temporal location as a precondition [21]. According to the client two types of scenarios are considered to be best suited

i. Current scenarios that describe the compulsory conditions of any applications.

ii. Future scenarios that describe the expected conditions that will take place in the context of use of software.

\subsection{Goal Driven and Decompression Technique}

In this technique communicating requirement collected by communications between different stakeholders for reaching final agreement on suggested requirement and also communication between different development team members. And finally evolving requirement can be get as the ability to manage the change in requirement effectively. Communication is a major issue and it becomes more complicated during the process of requirements rather than other risks [22]. In this technique language barrier can be dealt with by choosing a team leader from every site of developments, choosing of such personal should take into account his language, communication 
and technical capabilities as he is the one who will be the point of communication to other development members overseas travel cost issue also reduce by sending team member in such countries where labor cost is low such as India, China, Egypt etc [23].

\subsection{Project Management Models}

The distributed software development tools that are existing ones and are used as software development tools.

Table 1: Software Development Models

\begin{tabular}{|l|l|}
\hline \multicolumn{1}{|c|}{ Model } & \multicolumn{1}{c|}{ Research Study } \\
\hline $\begin{array}{l}\text { Project Management } \\
\text { Model [32] }\end{array}$ & $\begin{array}{l}\text { The project management model consists of } \\
\text { spiral type life cycle, UML and Object } \\
\text { oriented process. }\end{array}$ \\
\hline NEXT MOVE [33] & $\begin{array}{l}\text { Next Move is based upon collaboration of } \\
\text { team member, task coordination and plays } \\
\text { a significant role in monitoring and } \\
\text { communication. }\end{array}$ \\
\hline $\begin{array}{l}\text { Project Management } \\
\text { Framework [34] }\end{array}$ & $\begin{array}{l}\text { The project management framework is } \\
\text { used to make communication between } \\
\text { sites efficient. It was developed using } \\
\text { Microsoft project. }\end{array}$ \\
\hline TAPER [35] & $\begin{array}{l}\text { Taper can be referred as a framework for } \\
\text { development of good performance to } \\
\text { eliminate the challenges. }\end{array}$ \\
\hline CARMEL [36] & $\begin{array}{l}\text { CARMEL is a framework for managing } \\
\text { the attention regarding the communication } \\
\text { in context to meeting sessions. }\end{array}$ \\
\hline
\end{tabular}

Table 1 demonstrates the models that are used in software project management as a part of Global software development. The research is aimed at identifying the models that are effective specifically in GSD in context to distributed software development. There are many collaborative tools that are used for the coordination of a project and includes various frameworks that enhance the communication including the collaboration of the team. The analysis is done on various different models that can be used in Global software development to improve the collaboration between the team.

\section{RESEARCH METHODOLOGY}

In this research Qualitative method is used to analyze the data. Mainly, first objective is gathering requirements so there are many ways to do this like observation, interview, survey and case study. The major factor in requirement engineering is people involvement in the implementation of project. If we see it on the global scope, then there are many other constraints that do matter to get requirements like social media involvement to do interact with client to get requirements. Most of the work included the descriptive data, in which existing work was analyzed and from existing work different requirement gathering techniques were extracted. From previous contributions four techniques to gather requirements in software engineering were identified.

\subsection{Data Collection}

Data can be collected by scattering project team members in different countries that response back to the head office of the project for developing software. This technique is considered as a good approach to complete the gap of geographical location as well as temporal location. We collect data by using these following activities to make best scenario for requirement gathering [24]. In our research we have used qualitative technique to analyze the data. Because in which our first objective is gather requirements so there are many ways to do this like observation, interview, survey, case study

These are following activities must do when making scenarios:

- Creation of Language Extended Lexicon

- Validation of LEL through sessions

- Creation of Current Scenarios

- Validation of Current Scenarios through sessions

- Storage of LEL and Current Scenarios in the knowledge management repository

- Formal approval of LEL

- Formal approval of Current Scenarios

- Elicitation and analysis of business requirements

- Creation of a business requirements record

- Creation of a traceability matrix

- Validation of business requirements through sessions

- Formal approval of business requirements

- Creation of Future Scenarios

- Validation of Future Scenarios through sessions

- Storage of Future Scenarios in de knowledge management repository Formal approval of Future Scenarios

\section{ANALYSIS}

\subsection{Analysis between Elect Project Leader}

First one is Elect project leader in which just single person conduct requirements from the 
client as leader. It is very hazardous technique even though all system depends upon that single person [25]. The second one is Video Conferencing; it is better than first technique because in which all team members take part in requirement gathering from client through online video conference but it is also risky because its issue about network. If during conference internet signal goes to drop or missing the client voice, then due to it many problems can be occur like due to missing voice client tell important point but due to signals meeting members (developers side) could not listen that and miss that point same like as many issues can create in it. The third one is a sort of problem in specific context so its solution will be according to this problem was explain Interview through it project member do involve people and get requirements. Due to it we minimize the risk because in which client and developers do face to face interaction with each other. Fourth one is Facilitated Session in which large group is involve to gather requirements and its major benefit is also that if any member in missing then other will be evidence from that group of that conducted requirements. It is very fast and time saving technique [26].

\subsection{Analysis between Interview and Domain Analysis}

Designing interviews and domain analysis both are the key techniques that one can use in gathering requirements. We cannot rank both the techniques because both have its own importance. One technique can be used in gathering requirements from the stake holder while the latter can be used in interpretation of those requirements by the domain expert who will further elaborate the requirements to the developer. Both the discussed techniques can be used in their respective time with their respective importance.

\subsection{Analysis of Scenarios}

The data we collect is highly effective to answer our research question. Scenarios is mostly used in global software engineering because they used language extended lexicon model to communicate with different countries client it defines keywords which can easily understand to every client as well as developers and analysts. It also Improve quality of communication, the vocabulary of the domain throughout the entire software process. Both, Current Scenarios and Future Scenarios, also provided a communication medium, allowing a better understanding for the stakeholders, in addition those models are also written in the domain terminology. Such terminology is regular used by users and other human. It provides better knowledge of the problem domain, through scenarios that gave a proper view of the problem; the current behavior (Current Scenarios) and the planned behavior (Future Scenarios). The problems related to cultural and language barriers resolved through the LEL model, which unified the vocabulary for stakeholder's interaction, providing shared terms with a precise meaning. So, the data is helping to collect requirements without threats that highly map to our research question [27].

\subsection{Analysis between Scenarios and Goal Driven Technique}

The analysis between these two techniques are that Scenarios are best technique comparatively goal driven techniques we can also use LEL model to complete the communication gap between different people with separate language and geographical locations. It provides better knowledge to the problem. Scenarios collect requirements according to current problem and also design future scenarios if required. It's also reducing the ambiguity and lack of clarity in the specification during requirement gathering. It also improves trust between clients and developer rather than other technique it does not ensure that they provide future changing if required. Goal driven is also a good approach but it is limited scope area it uses flow diagrams to show requirements as compared to scenarios it is better approach because it collects all requirements using use cases in which every user requirement or stakeholder's requirement noted until system develops. If stakeholders want to add some new requirement, then they direct communicate with analyst who collect requirement but in goal driven technique stakeholders do not interact direct with analyst they inform local manager who manage whole system he check either this new requirement is valid or not if valid they pass otherwise not pass to analyst I think this is lack of trust may be requirement is valid or he does not provide software according to stakeholder's desire. So, we suggest after reading research papers 
examples that Scenarios have good life cycle to collect requirements as compared to goal driven technique.

\section{RESULTSAND DISCUSSION}

The research carries significant importance as it is covering the vast subject which can be investigated in a better way in interview sessions. The challenged that were encountered included. The difficult task in software development is to define the requirements. The major problem in requirement engineering starts from this phase. Basically, the requirements are not clear and client cannot define the problems and translating the requirements according to the technical perspective from a business one is difficult. There is a need of special skills to gather the requirements. Requirement gathering skills can be learnt from some particular workshops which can help the client in demonstrating requirements. Therefore, there is a need of emphasizing the RE practices in context to the organizations that will help to increase the software development quality. The quality attributes include defining that whether the requirements are achievable, defined properly, detailed and that are at the right level of detail [28].

The results that were generated after the experimental procedure concluded that the variation in $\mathrm{RE}$ is solely related to the process being adopted. The process could be among waterfall, agile in context to RE execution. The quality attributes are examined and validated in various cases along with emphasizing on the role of participant while focusing on the quality of $\mathrm{RE}$ [29]. The findings that has been derived as a success factors of RE include:

i. Inspiration, willingness of participant

ii. Usability of RE process

iii. Enhancement of activities.

iv. Implementing the techniques and strategies

In short on the basis of the findings generated, the authors of the research concluded that the basic foundation of RE Processes is majorly dependent on three dimensions including human dimensions, infrastructure dimensions and characteristics of RE processes. The quality of these RE processes is therefore directly linked to these three dimensions mentioned above. There is a need to pinpoint the situation where the project becomes global for the particular organization. Along with that here is a need to identify the strategies adopted by organization when RE processes are to be handled [30].

The major factor in requirement engineering is people involvement in the implementation of project. If we see it on the global scope then there are many other constraints do matter to get requirements like social media involvement to do interact with client to get requirements. In our research we do Case Study. Most of the work included the descriptive data, we did read existing work and from existing work we extract different requirement gathering techniques.

First one is Elect project leader in which just single person conduct requirements from the client as leader. It is very risky technique even though all system depends upon that single person. The second one is Video Conferencing; it is better than first technique because in which all team members take part in requirement gathering from client through online video conference but it is also risky because its issue about network. If during conference internet signal goes to drop or missing the client voice then due to it many problems can be occur like due to missing voice client tell important point but due to signals meeting members (developers side) could not listen that and miss that point same like as many issues can create in it. The third one is a sort of problem in specific context so its solution will be according to this problem was explain Interview through it project member do involve people and get requirements. Due to it we minimize the risk because in which client and developers do face to face interaction with each other. Fourth one is Facilitated Session in which large group is involve to gather requirements and its major benefit is also that if any member in missing then other will be evidence from that group of that conducted requirements. It is very fast and time saving technique [31].

From all these four techniques Interview and Facilitated Session are best because both are less time consuming and faster than other two techniques. But from these two one of it is widely used technique in global requirement engineering which is Interview because in which client and developer directly interact with each other and can easily resolve problems and can 
ask question regarding the project. As well as due to it we can judge market trends and people need on global scope. Facilitated session is not a common technique and most of the people did not know about it so it will also not use widely in global software requirements engineering

\section{CONCLUSION AND FUTURE DIRECTIONS}

Global software development is increasingly day by day. For it requirement gathering is an important factor in Global Software Engineering. So, Requirement gathering technique becomes a major problem in this region. We conduct literature review to find out the best techniques used in GSE. We do case study in our research to collect data according to our research question. After reading different papers on this domain we finalize that there are two or more techniques that are suitable to collect requirements. No one specific technique is used to collect requirements because every project has different domain as well as different stakeholder's demand like some companies prefer to communicate through video conferencing, some prefer to arrange proper meeting for telling their requirements. Some stakeholders prefer to arrange interviews. Other one has appreciated scenario-based method to collect requirements. To consider all these situations, we used qualitative analysis data approach to conclude our proposed solution is that every technique has their own advantages as well as characteristics we can select requirement gathering technique straight away when we have come to know about project domain also stakeholders demand.

For future work there is a need of conducting more surveys to gather the feedback of various audiences that are working on RE in context to GRE Projects. The research can be done on more specific area of RE according to the view point of various authors including the factors effecting the RE practices while in GRE Projects. Highlighting the risks factors when not adopting transparency while working on a GRE project. The research can be done on development phase or testing phase in the field of GRE. We hope this research will be an inspiration to the further work in the GRE field.

\section{Conflict of Interest}

On behalf of all authors, the corresponding author states that there is no conflict of interest.

\section{References}

[1] Zanoni, R. \& Audy, J. L. N. 2012 "Project management model for a physically distributed software development environment" Proc. 36th Annual Hawaii International Conference on System Sciences, 8pp.

[2] Mak, D. K. M., Kruchten, P. B. 2017 "NextMove: A Framework for Distributed Task Coordination" Proc. 18th Australian Software Engineering Conference ASWEC 2007, 399408.

[3] Ghosh, P. P. \& Varghese, J. C. 2014 "Globally distributed product development using a new project management framework" International Journal of Project Management, Elsevier.

[4] Hofner, G. \& Mani, V. S. 2015 “TAPER: A generic framework for establishing an offshore development center" Proc. Second IEEE International Conference on Global Software Engineering ICGSE 2007, 162-172.

[5] Lee-Kelley, L. \& Sankey, T. 2017 "Global virtual teams for value creation and project success: A case study" International Journal of Project Management, Elsevier.

[6] Hussain, S. Aliyu, M. Barakat,F. Maki, "Assessing and testing the usability of student portal," in Proc.3rd Int.Conf. Applied sciences and technology,ICAST,Vol.1,2018,pp.20-51.

[7] Ali, M. qasim, Nargis, Noshaba, Yasmeen, Rehana, Iqba, Zafar "ICT Use for Effective Teaching-Learning Process in Secondary Schools in Punjab Province", Vol 4,pp.7

[8] H.Gull, S.Z.Iqbal, M.Saqib, "Usability Evaluation of an educational website in Saudia Arabia," Vawkum transactions on Computer science, vol.1,no.2, 2015, pp1-9.

[9] L.Hasan, "Evaluating the Usability of educational websites based on students preferences of design characteristics," International Arab Journal of etechnology,vol.3,no.3,2014, pp.15. 
[10] M.El Tahir Osman, "Educational Portal i n O m a n: Tow ard a connected community"AMARABAC, Journal of American Arabic Academy for Sciences and Technology, www.amarabac.com Volume 1, Number 2, (2010), PP. 101-111

[11] Haas,Ludwing "Schola Europea--The European School : The Fifteen-Nation School in Luxemburg. The Pedagogical Mini-Europe",in European Education, Vol 36,no 3,pp.77-86,2004

[12] M. J. S. Asiri, R.Mahmud, K.A.Bakar, A.F .M.Ayub "Factors Influencing the Use of Learning Management System in Saudi Arabian Higher Education: A Theoretical Framework", Vol. 2, No. 2; June 2012.

[13] Butt, N., Warraich, N. and Tahira, M. (2019), "Development level of electronic government services", Global Knowledge, Memory and Communication, Vol. 68 No. 1/2, pp. 33-46.

[14] S. Scott, U.Kokil,"Usability Testing of a School Website using Qualitative Approach,", in Int. Conf.Human Computer Interaction Theory and Applications, Kannesaw,(visigrapp), vol $.2,2017$,pp.55-64.

[15] S.Roy,P.K.Pattnaik, R.Mall, "A quantitative approach to evaluate usability of academic websites based on human perception,",Egyptian Informatics Journal, Vol.15,no.3 pp.9, 2014.

[16] M. A. Jabar, U.A. Usman, A. Awal, "Assessing the Usability of University Websites from Users' Perspective," Australian Journal of Basic and Applied Sciences, no. 10, pp. 98-111, 2013.

[17] A. Hussain, S. Aliyu, M. Barakat,F. Maki, "Assessing and testing the usability of student portal," in Proc.3rd Int.Conf.Applied $\mathrm{s}$ c i e n c e s a n d technology,ICAST,Vol.1,2018,pp.20-51.

[18] H. Al-Dossari, "A Heuristic based approach for usability evaluation of e-portal systems," International Journal of Computer Science \& Information Technology (IJCSIT) ,Vol. 9, no. 3,2017, pp.1-16.

[19] H.Gull,S.Z.Iqbal,M.Saqib,"Usability
Evaluation of an educational website in saudia arabia," Vawkum transactions on Computer science, vol.1,no.2,2015, pp. 1-9.

[20] L.Hasan, "Evaluating the usability of educational websites based on students preferences of design characteristics," International Arab Journal of etechnology,vol.3,no.3,2014, pp.15.

[21] S.Scott,U.Kokil,"Usability Testing of a School Website using Qualitative Approach," ,in Int. Conf.Human Computer Interaction Theory and Applications,Kannesaw,(visigrapp),vol $.2,2017$, pp.55-64.

[22] S.Roy,P.K.Pattnaik, R.Mall, "A quantitative approach to evaluate usability of academic websites based on human perception,",Egyptian Informatics Journal, Vol.15,no.3 pp.9, 2014.

[23] M. A. Jabar, U.A. Usman, A. Awal, "Assessing The Usability Of University Websites From Users' Perspective," Australian Journal of Basic and Applied Sciences, no. 10, pp. 98-111, 2013.

[24] M. Iqbal, N.F.Warraich, "Usability evaluation of an academic library website: A case of the University of the Punjab," Pakistan Journal of Library \& Information Science, vol.13,no.1, pp. 1-11, 2012.

[25] F.Oliha, "Web portal usability among nigerian university students," Nigerian Journal of Technology (nijotech), vol. 33, no. 2, pp. 199206, 2014.

[26] D. P.Vukovac , K.B. Kirinic, "A comparison of e-learning sysems for usability evaluation," in daam internationl scientific book, Daam international Vienna, 2010, pp. 271-288.

[27] A. Rukshan, "A quantitative approach to usabiity evaluation of websites," in Proceedings of Advances in computing technology ,London,United Kingdom,2011.

[28] Y. S. Ryu, "Development of Usability Questionnaires for Electronic Mobile Products and Decision Making Methods," Faculty of Virginia Polytechnic Institute and State University, Blacksburg, Virginia,Vol.2,no.1, 
2005.

[29] L.A.Daher, I. Elkabani, "Usability evaluation of some lebanese universities web portals," in the 13th international Arab conference on Information Technology, Lebanon, pp.10-13,2013.

[30] S.K.Dubey,A. Rana "Analytical Roadmap to Usability Definitions and Decompositions," international journal of Engineering Sciencee and Technology, vol.2, no. 9 , p. $8,2010$.
[31] S.Qadar, A.Moazzam,N.Ansari, "E-Tax Website Quality:An Evaluation Framework," Pakistan Journal of Information Management and Libraries, vol. 16, pp. 1-11, 2015. 
